\title{
LOEWY CHAINS AND UNIFORM SPLITTING OF LATTICES
}

\section{AILEEN HOSTINSKY}

1. Introduction. This paper is devoted to a proof of a theorem on the existence of a Loewy $\eta$-chain as a sufficient condition for uniform splitting. The system considered is a complete modular lattice $L$ (with the usual binary operations denoted by $p+q$ and $p q$ for $p$ and $q$ in $L$ ) satisfying the additional Property (*):a $\sum_{\alpha} p_{\alpha}=\sum_{\alpha} a p_{\alpha}$ for $a$ and the ascending chain $p_{\alpha}$ elements of $L$.

For a complete statement of results made use of here, the reader is referred to a previous paper [1]. For convenience some basic definitions from that paper are listed in the remainder of this section. A single-valued transformation $\eta$ is a homomorphism of $p / p^{\prime}$ in $L$ upon $q / q^{\prime}$ in $K$ (a system satisfying the same postulates as $L$, or $L$ itself) if $\eta$ maps $x / p^{\prime}$ for every $x$ satisfying $p^{\prime} \leqq x \leqq p$ upon $x \eta / q^{\prime}$, where $q^{\prime}$ $\leqq x \eta \leqq q$, so that the following requirements hold:

(1) $\left(\sum_{x} x\right) \eta=\sum_{x}(x \eta)$.

(2) If $q^{\prime} \leqq z \leqq x \eta$, then there exists an element $z^{\prime}$ with $p^{\prime} \leqq z^{\prime} \leqq x$ such that $z^{\prime} \eta=z$.

(3) If $x \eta=y \eta$, then there exist elements $x^{\prime}$ and $y^{\prime}\left(p^{\prime} \leqq x^{\prime} \leqq p\right.$ and $\left.p^{\prime} \leqq y^{\prime} \leqq p\right)$ such that $x+x^{\prime}=y+y^{\prime}$ and $x^{\prime} \eta=y^{\prime} \eta=q^{\prime}$.

A homomorphism $\eta$ of $p / 0$ upon $p \eta / 0$ is said to be an endomorphism of $p / 0$ if $p \eta \leqq p$. The kernel $k(\eta)$ of this endomorphism is the sum of all elements which $\eta$ maps upon 0 , and the radical $r(\eta)$ is defined as $\sum_{i=1}^{\infty} k\left(\eta^{i}\right) . x$ is $\eta$-admissible if $x \eta \leqq x$. The usual homomorphism and isomorphism theorems hold.

The element $c$ is a complement of the endomorphism $\eta$ of $p / 0$ if $p=r(\eta)+c, c r(\eta)=0$ (that is, $p=c \oplus r(\eta)$ ), and $c \eta=c$. If there exists a complement of $\eta$, then $\eta$ is a splitting endomorphism of $p / 0$. If $\eta$ induces a splitting endomorphism of $x / 0$ for every $\eta$-admissible $x, \eta$ is a uniformly splitting endomorphism of $p / 0$.

2. Proof of the theorem. Several definitions and lemmas are required for the proof of the following theorem.

THEOREM. The endomorphism $\eta$ of $p / 0$ is a uniformly splitting endomorphism if there exists a Loewy $\eta$-chain the sum of which is $p$.

Definition 1. An $\eta$-admissible element $q$ is minimal $\eta$-admissible over $r$ if $r<s \leqq q$, for $\eta$-admissible $s$, implies that $q=s$.

Definition 2. Elements $a_{i}(i=0,1,2, \cdots)$ form a Loewy $\eta$-chain

\footnotetext{
Presented to the Society, April 25, 1953; received by the editors July 3, 1953.
} 
if $a_{0}=0$ and every $a_{i}$ with $i>0$ is the sum of elements which are minimal $\eta$-admissible over $a_{i-1}$.

It is clear from the definition that the chain of the $a_{i}$ is ascending and that every $a_{i}$ is $\eta$-admissible.

LemMa 1. Let $\eta$ be a homomorphism of $m / r$ upon $m \eta / r$ and let $m$ be minimal $\eta$-admissible over $r$. Then either $m \eta=r$ or $m \eta$ is minimal $\eta$-admissible over $r$. Furthermore, if $m$ is a sum of elements minimal $\eta$-admissible over $r$, then $m \eta=r$ or $m \eta$ is a sum of elements minimal $\eta$-admissible over $r$.

Proof. Suppose that $m \eta \neq r$ and that there exists an $\eta$-admissible $s$ such that $r<s<m \eta$. Since $m \eta \leqq m$, the second supposition contradicts the fact that $m$ is minimal $\eta$-admissible. Thus $m \eta$ is minimal $\eta$-admissible over $r$. Moreover, if $m=\sum_{\alpha} m_{\alpha},\left(\sum_{\alpha} m_{\alpha}\right) \eta=\sum_{\alpha}\left(m_{\alpha} \eta\right)$, and the preceding result gives the second conclusion.

Lemma 2. If $z \leqq y \leqq x$, if $x$ is the sum of elements minimal $\eta$-admissible over $z$ (and included in $x$ ), and if $y \eta \leqq y$, then there exists $t^{*}$ such that $x=y+t^{*}$ and $y t^{*}=z$, and $y$ is equal to $z$ or is the sum of elements minimal $\eta$-admissible over $z$ (and included in $y$ ).

Proof. Let $A=\{t \in L \mid t \eta \leqq t, t \leqq x$, and $t y=z\}$, let $B=\left\{t_{\alpha}\right\}$ be a simply ordered subset of $A$, and let $t^{\prime}=\sum_{\alpha} t_{\alpha}$. Then $t^{\prime} \eta \leqq t^{\prime}, t_{\alpha} \leqq t^{\prime} \leqq x$ for every $\alpha$ and $y t^{\prime}=y \sum_{\alpha} t_{\alpha}=\sum_{\alpha} y t_{\alpha}=z$, by Property $\left({ }^{*}\right)$ and the definition of $A$. Thus the maximal principle is applicable and asserts the existence of a maximal element $t^{*}$ in $A$, with $y t^{*}=z$.

Let $x=\sum_{m} m$, the sum of elements minimal $\eta$-admissible over $z$, and consider any $m$. Then $z \leqq m\left(y+t^{*}\right) \leqq m$, and hence either $m$ $=m\left(y+t^{*}\right)$ or $m\left(y+t^{*}\right)=z$. If the latter were true, by the modular law, $y\left(t^{*}+m\right)=y\left(t^{*}+m\right)\left(y+t^{*}\right) \leqq\left(t^{*}+m\right)\left(y+t^{*}\right)=t^{*}+m\left(y+t^{*}\right)=t^{*}$ $+z=t^{*}$. Hence $z \leqq y\left(t^{*}+m\right) \leqq y t^{*}=z$, and $t^{*}+m$ is in $A$. By the maximal property of $t^{*}, t^{*} \nless t^{*}+m$, and hence $t^{*}=t^{*}+m$ and $m \leqq t^{*}$. But then $m=m t^{*}=z$. In this case $x=\sum_{m} m=z$, and thus $y=x$, $t^{*}=z$, and the lemma is proved.

If $m=m\left(y+t^{*}\right), m \leqq y+t^{*}$. Consequently $x=\sum_{m} m \leqq y+t^{*} \leqq x$, or $x=y+t^{*}$. The last equation implies the existence of an isomorphism $\left[1\right.$, p. 334, Theorem 3] of $x / t^{*}$ upon $y / z$. Hence, by an obvious generalization of $[1$, p. 333 , Theorem 1$]$, there is a homomorphism of $x / z$ upon $y / z$. Thus, by Lemma $1, y$ is the sum of elements minimal $\eta$-admissible over $z$ (or $z$ ).

LEMma 3. If elements $a_{i} \leqq p$ form a Loewy $\eta$-chain, then $a_{i} r(\eta)$ $=a_{i} k\left(\eta^{i}\right)$ for all $i$. 
Proof. The lemma is proved by induction with respect to $i$. Since $a_{0}=0$, the assertion is true for subscript 0 . As induction hypothesis, it will be assumed that $a_{i-1} r(\eta)=a_{i-1} k\left(\eta^{i-1}\right)$.

Let $Q$ be the set of elements $q$, with $a_{i-1}<q \leqq a_{i-1}+a_{i} r(\eta)$ $=\sum_{n=1}^{\infty}\left(a_{i-1}+a_{i} k\left(\eta^{n}\right)\right)$, which are minimal $\eta$-admissible over $a_{i-1}$. By Property (*)

$$
q=q \sum_{n=1}^{\infty}\left(a_{i-1}+a_{i} k\left(\eta^{n}\right)\right)=\sum_{n=1}^{\infty} q\left(a_{i-1}+a_{i} k\left(\eta^{n}\right)\right) .
$$

Furthermore, the modular law shows that $q \geqq q\left(a_{i-1}+a_{i} k\left(\eta^{n}\right)\right)=a_{i-1}$ $+q a_{i} k\left(\eta^{n}\right)=a_{i-1}+q k\left(\eta^{n}\right)=q\left(a_{i-1}+k\left(\eta^{n}\right)\right) \geqq a_{i-1}$. Thus

$$
q=\sum_{n=1}^{\infty} q\left(a_{i-1}+k\left(\eta^{n}\right)\right) \quad \text { and } \quad a_{i-1} \leqq q\left(a_{i-1}+k\left(\eta^{n}\right)\right) \leqq q .
$$

If $q\left(a_{i-1}+k\left(\eta^{n}\right)\right)=a_{i-1}$ for every $n$, then $q=\sum_{n=1}^{\infty} q\left(a_{i-1}+k\left(\eta^{n}\right)\right)=a_{i-1}$. Hence there exists an $m=m(q)$ such that $a_{i-1}<q\left(a_{i-1}+k\left(\eta^{m}\right)\right) \leqq q$. Since $q$ is minimal $\eta$-admissible over $a_{i-1}, q=q\left(a_{i-1}+k\left(\eta^{m}\right)\right)=a_{i-1}$ $+q k\left(\eta^{m}\right)$. Thus $q \eta^{m}=a_{i-1} \eta^{m} \leqq a_{i-1}$.

Since $a_{i-1} \leqq a_{i-1}+q \eta \leqq q$ and since $a_{i-1}+q \eta$ is $\eta$-admissible, either $a_{i-1}=a_{i-1}+q \eta$ or $q=a_{i-1}+q \eta$. If $q=a_{i-1}+q \eta$, then

$$
a_{i-1}<q=a_{i-1}+q \eta=\cdots=a_{i-1}+q \eta^{m}=a_{i-1},
$$

since $q=a_{i-1}+\left(a_{i-1}+q \eta\right) \eta=a_{i-1}+a_{i-1} \eta+q \eta^{2}=a_{i-1}+q \eta^{2}=\cdots$ and since $q \eta^{m} \leqq a_{i-1}$. This impossibility shows that the other alternative, $a_{i-1}=a_{i-1}+q \eta$, must hold. Therefore $q \eta \leqq a_{i-1}$ for every $q$.

By Lemma 2, $a_{i-1}+a_{i} r(\eta)=\sum_{q} q$, the sum of elements minimal $\eta$-admissible over $a_{i-1}$. Since every $q \eta \leqq a_{i-1}$,

$$
\sum_{q}(q \eta)=\left(\sum_{q} q\right) \eta=\left(a_{i-1}+a_{i} r(\eta)\right) \eta \leqq a_{i-1} .
$$

Moreover, $\left(a_{i} r(\eta)\right) \eta \leqq\left(a_{i-1}+a_{i} r(\eta)\right) \eta \leqq a_{i-1}, \quad$ and $\left(a_{i} r(\eta)\right) \eta \leqq a_{i-1} r(\eta)$ $=a_{i-1} k\left(\eta^{i-1}\right) \leqq k\left(\eta^{i-1}\right)$, by the induction hypothesis. Therefore $\left[\left(a_{i} r(\eta)\right) \eta\right] \eta^{i-1}=\left(a_{i} r(\eta)\right) \eta^{i}=0$, and this fact implies that $a_{i} r(\eta) \leqq k\left(\eta^{i}\right)$. Hence $a_{i} k\left(\eta^{i}\right)=a_{i} r(\eta)$, and the lemma is proved.

Leмma 4. If the $a_{i}$ form a Loewy $\eta$-chain, then $a_{i}=a_{i} \eta^{j}+a_{i} r(\eta)$ for every $i$ and $j$.

Proof. The proof is by induction with respect to $i$. The assertion is trivially true for subscript 0 . Assume, as induction hypothesis, that for $0<i, a_{i-1}=a_{i-1} \eta^{j}+a_{i-1} r(\eta)$ for every $j$.

Let $q$ be minimal $\eta$-admissible over $a_{i-1}$ such that $a_{i-1}<q \leqq a_{i}$. 
Two cases are to be considered.

Case 1. $q \eta \leqq a_{i-1}$. By Lemma 3 and the induction hypothesis

$$
q \eta \leqq a_{i-1}=a_{i-1} \eta+a_{i-1} k\left(\eta^{i-1}\right)=a_{i-1} \eta^{j}+a_{i-1} k\left(\eta^{i-1}\right) .
$$

Since $a_{i-1}<q$, it follows that $q \eta^{i}=a_{i-1} \eta^{i}$. It is a consequence of (1) also that $a_{i-1} \eta^{i}=a_{i-1} \eta^{j+i}$ for all $j$ and hence $q \eta^{i}=\left(a_{i-1} \eta^{j}\right) \eta^{i}$ for all $j$. Since $\eta^{i}$ is an endomorphism of $p / 0$, the last equality shows that $q+k\left(\eta^{i}\right)$ $=a_{i-1} \eta^{j}+k\left(\eta^{i}\right)$. Thus

$$
q=q a_{i} \leqq\left(a_{i-1} \eta^{j}+k\left(\eta^{i}\right)\right) a_{i}=a_{i-1} \eta^{j}+a_{i} k\left(\eta^{i}\right) \leqq a_{i} \eta^{j}+a_{i} r(\eta) \leqq a_{i} .
$$

Case 2. $q \eta a_{i-1}$. In this case $a_{i-1}<a_{i-1}+q \eta \leqq q$, and $a_{i-1}+q \eta$ is $\eta$-admissible. Hence, by the minimal property of $q, q=a_{i-1}+q \eta$. The induction hypothesis then implies that, for all $j$,

$$
\begin{aligned}
q & =a_{i-1}+q \eta=a_{i-1}+q \eta^{2}=\cdots=a_{i-1}+q \eta^{j} \\
& =a_{i-1} \eta^{j}+a_{i-1} r(\eta)+q \eta^{j} \\
& \leqq a_{i} \eta^{i}+a_{i} r(\eta)+q \eta^{i}=a_{i} \eta^{j}+a_{i} r(\eta) \leqq a_{i} .
\end{aligned}
$$

Since $a_{i}=\sum_{q} q$, in both cases $a_{i}=a_{i} \eta^{j}+a_{i} r(\eta)$ for every $i$ and $j$.

Lemma 5. If the $a_{i}$ form a Loewy $\eta$-chain, then $a_{i}=a_{i} \eta^{i} \oplus a_{i} r(\eta)$ and $a_{i} \eta^{i}=a_{i} \eta^{i+1}$ for every $i$.

Proof. By Lemma $4, a_{i}=a_{i} \eta^{j}+a_{i} r(\eta)$ for all $i$ and $j$. Hence for $j=1$, by Lemma $3, a_{i}=a_{i} \eta+a_{i} k\left(\eta^{i}\right)$, for all $i$. As a result, $a_{i} \eta^{i}=a_{i} \eta^{i+1}$, and the chain

$$
a_{i} \geqq a_{i} \eta \geqq \cdots \geqq a_{i} \eta^{i}=a_{i} \eta^{i+1}=\cdots
$$

is finite. By Lemma 3 , the chain

$$
a_{i} k(\eta) \leqq a_{i} k\left(\eta^{2}\right) \leqq \cdots \leqq a_{i} k\left(\eta^{i}\right)=a_{i} k\left(\eta^{i+1}\right)=\cdots=a_{i} r(\eta)
$$

is also finite, where the terms are the kernels of the respective endomorphisms induced in $a_{i}$. [1, p. 339, Theorem 3] now gives the result $a_{i}=a_{i} \eta^{i} \oplus a_{i} r(\eta)$, for $i=0,1,2, \cdots$.

Lemma 6. An endomorphism $\eta$ of $p / 0$ is a splitting endomorphism of $p / 0$ if there exists a Loewy $\eta$-chain the sum of which is $p$.

Proof. Let $a_{0} \leqq a_{1} \leqq \cdots$ be the Loewy $\eta$-chain. By Lemma 5 , for each $i, a_{i}=a_{i} \eta^{i} \oplus a_{i} r(\eta)$. By hypothesis, it follows that

$$
p=\sum_{i=0}^{\infty} a_{i}=\sum_{i=0}^{\infty}\left[a_{i} \eta^{i}+a_{i} r(\eta)\right]=\sum_{i=0}^{\infty} a_{i} \eta^{i}+\sum_{i=0}^{\infty} a_{i} r(\eta) .
$$

Let $\sum_{i=0}^{\infty} a_{i} \eta^{i}=c$. By Property $\left(^{*}\right)$, 


$$
\sum_{i=0}^{\infty} a_{i} r(\eta)=r(\eta) \sum_{i=0}^{\infty} a_{i}=r(\eta) p=r(\eta) .
$$

Hence $p=c+r(\eta)$.

By Lemma 5, $\left(a_{i} \eta^{i}\right) \eta=a_{i} \eta^{i}$ and $0=\left(a_{i} \eta^{i}\right)\left(a_{i} r(\eta)\right)=\left(a_{i} \eta^{i}\right) r(\eta)$ for all $i$. Hence $c \eta=c$, and

$$
\operatorname{cr}(\eta)=r(\eta) \sum_{i=0}^{\infty} a_{i} \eta^{i}=\sum_{i=0}^{\infty} r(\eta) a_{i} \eta^{i}=0 .
$$

Thus there exists a complement $c$, and $\eta$ is a splitting endomorphism of $p / 0$.

Proof of THE THEOREM. Let $a_{0} \leqq a_{1} \leqq \cdots$ be the Loewy $\eta$-chain and let $s$ be $\eta$-admissible and included in $p$. Then the $s a_{i}$ form an ascending chain of $\eta$-admissible elements, and, by Property $\left(^{*}\right)$,

$$
\sum_{i=0}^{\infty} s a_{i}=s \sum_{i=0}^{\infty} a_{i}=s p=s .
$$

Since $a_{i-1} \leqq a_{i-1}+s a_{i} \leqq a_{i}=\sum_{q} q$, where $q$ is minimal $\eta$-admissible over $a_{i-1}$, it follows by use of Lemma 2 that $a_{i-1}+s a_{i}$ is the sum of elements minimal $\eta$-admissible over $a_{i-1}$. A basic isomorphism theorem gives the existence of an isomorphism of $\left(a_{i-1}+s a_{i}\right) / a_{i-1}$ onto $s a_{i} /\left(s a_{i}\right)\left(a_{i-1}\right)=s a_{i} / s a_{i-1}$. Thus it can be concluded that $s a_{i}$ is the sum of elements which are minimal $\eta$-admissible over $s a_{i-1}$. Thus the $s a_{i}$ form an ascending Loewy $\eta$-chain, the sum of which is $s$, and, by Lemma 6 , the endomorphism induced by $\eta$ in $s / 0$ is a splitting endomorphism of $s / 0$. Hence $\eta$ is a uniformly splitting endomorphism of $p / 0$.

\section{REFERENCE}

1. L. A. Hostinsky, Endomorphisms of lattices, Duke Math. J. vol. 18 (1951) pp. 331-342.

The Pennsylvania State University 